\title{
IDADE E CRESCIMENTO DE GYMNOGEOPHAGUS LACUSTRIS REIS \& MALABARBA, UM CICHLIDAE ENDÊMICO DA BACIA HIDROGRÁFICA DO RIO TRAMANDAÍ, RIO GRANDE DO SUL, BRASIL
}

\author{
Sandra Maria Hartz ${ }^{1}$ \\ Willi Bruschi Junior ${ }^{1}$ \\ Márcio Vanini Formehl ${ }^{1,2}$
}

\begin{abstract}
AgE AND GROWTH OF GYMNOGEOPH.AGUS LACUSTRIS REIS \& MALABARBA, an ENDEMic Cichlidae of Tramandai River basin (Rio Grande do Sul, Brazil). In the present study it was examined the age and growth of Gymnogeophagus lacustris Reis \& Malabarba, 1988 in Caconde lagoon (Tramandaí River Basin). The specimens (1080 males and 267 females) were collected monthly, between July 1992 and June 1993. The growth curve in total length was obtained by von Bertalanffy expression and the weight curve through the dedutive method supported by the mathematical expressions of the length growth curve and the length/weight relationship. Formation of the annulli in scales ocurred between winter and spring, and the first anual ring appears when the animals are aproximately 1.5 years old. $L \propto$ and $W \propto$ obtained for males and females, were $206.6 \mathrm{~mm}$ and $157.3 \mathrm{~g}$ and $120.2 \mathrm{~mm}$ and $30.2 \mathrm{~g}$, respectively, demonstrating a characteristic sexual dimorfism for the species.

KEY WORDS. Gymnogeophagus, growth, coastal lagoon, Rio Grande do Sul, Brazil
\end{abstract}

As espécies do gênero Gymnogeophagus Ribeiro, 1918 apresentam, como característica sinapomórfica, um espinho no topo do primeiro pterigióforo dorsal, esta sendo única entre os ciclídeos neotropicais (REIS \& MALABARBA 1988). Seus representantes encontram-se distribuidos geograficamente ao longo das bacias do Prata até os sistemas de rios e lagoas costeiras nos estados brasileiros de Santa Catarina e Rio Grande do Sul e norte do Uruguai.

A região do litoral norte do Estado do Rio Grande do Sul possui uma extensa área recoberta com corpos de água de diversos tipos (lagoas de água-doce, lagunas, estuário e rios). Esta diversidade hídrica compõe a bacia hidrográfica do rio Tramandaí, a qual possui uma riqueza considerável de peixes, com alguns endemismos. Entre estas espécies endêmicas destaca-se o cará Gymnogeophagus lacustris Reis \& Malabarba, 1988 (MALABARBA \& IsAIA 1992). Esta fauna vendo sendo estudada, nestes últimos anos, quanto aos seus aspectos ecológicos e biológicos, sendo que, em um destes estudos, encontrou-se uma abundante população dessa

1) Centro de Ecologia, Universidade Federal do Rio Grande do Sul. Caixa Postal 15.007. 91.501-970 Porto Alegre, Rio Grande do Sul.

E-mail: hartzsm@ecologia.ufrgs.br

2) Bolsista de Iniciação Científica do Programa PIBIC/CNPq/UFRGS.

Revta bras. Zool. 15 (3): 605 - 612, 1998 
espécie numa lagoa isolada de água doce da região (Lagoa Caconde), constituindose numa das espécies dominantes da comunidade de peixes daquele local. Por ser, talvez, uma espécie descrita há pouco tempo e endêmica, não há nenhum trabalho realizado em outras localidades que aborde seus aspectos bioecológicos.

Estimativas dos parâmetros de crescimento das populações de peixes são de importância fundamental, não apenas para o entendimento do seu ciclo de vida, como também para o manejo destes recursos pesqueiros (LIZAMA \& VAZZOLER 1993). O presente trabalho tem por objetivos determinar a época de formação dos anéis etários, as curvas de crescimento em comprimento e peso e a relação peso $\mathrm{x}$ comprimento para machos e fêmeas de Gymnogeophagus lacustris da Lagoa Caconde.

\section{MATERIAL E MÉTODOS}

A lagoa Caconde localiza-se no Município de Osório (2952’ $\left.\mathrm{S}, 50^{\circ} 12^{\prime} \mathrm{W}\right)$, ocupando uma área de $3,5 \mathrm{~km}^{2}$. Apresenta um formato cordiforme, profundidades máxima de $4 \mathrm{~m}$ e média de 1,6 $\mathrm{m}$. Suas margens estão densamente ocupadas por macrófitas aquáticas, com a predominância de Scirpus californicus (C.A. Meyer) Steud, conhecida na região como junco. As características físicas e químicas da água são fortemente influencidas pela sazonalidade climática e pela morfologia da lagoa, cujo regime de circulação é polimítico, devido à grande influência do vento na região.

Um total de 1.347 exemplares (267 fêmeas e 1.080 machos) foram coletados com redes de espera de malhas variando de 12 a $35 \mathrm{~mm}$ entre-nós adjacentes e com uma rede de arrasto de margem (picaré), durante os meses de julho/92 a junho/93, mensalmente, em três locais distintos da lagoa. De cada animal foram registrados os dados de comprimento total (Lt), em mm, peso total (Wt), em g e sexo. Foram selecionados cerca de 30 animais (de tamanhos variados), a cada mês, para a retirada das escamas. Estas (de 5 a 6 ) foram retiradas do lado esquerdo do animal, na região anterodorsal, entre a linha lateral e a nadadeira dorsal (região de melhor leitura), e acondicionadas em álcool $70 \%$. Para cada exemplar foi anotado o número de anéis presentes nas escamas através de leitura em estereomicroscópio.

A época de formação do anel etário foi obtida plotando-se os valores médios dos comprimentos totais dos animais com um mesmo número de anéis em relação às estações do ano (trimestres). Para a análise das curvas de crescimento em comprimento foi utilizada a expressão de von Bertalanffy (BERTALANFFY 1938), após constatação de linearidade através da transformação de Ford-Walford (WALFORD 1946), considerando-se o comprimento ao nascer desprezivel $\left(t_{0}=0\right)$. A idade estimada de formação do primeiro anel etário $\left(t_{1}\right)$ foi estabelecida através da metodologia descrita em SANTOS (1978). A relação peso total x comprimento total foi representada pela expressão: $W t=\phi L t^{\theta}$, onde: $(\phi)$ fator de condição e $(\theta)$ constante relacionada ao tipo de crescimento (LE CREN 1951). As curvas de crescimento em peso foram derivadas das relações peso $\mathrm{x}$ comprimento e das curvas de crescimento em comprimento.

Revta bras. Zool. 15 (3): 605 - 612, 1998 


\section{RESULTADOS}

A figura 1 apresenta a distribuição das frequeências de captura das classes de comprimento total (em $20 \mathrm{~mm}$ ) para machos e fêmeas. As maiores fêmeas capturadas alcançaram comprimentos entre $120-140 \mathrm{~mm}$, enquanto encontrou-se machos na classe de $180-200 \mathrm{~mm}$, indicando um dimorfismo sexual acentuado de tamanho. Diferenças também foram obtidas quanto às classes modais $(120-140 \mathrm{~mm}$, para machos, e 80-100 mm, para fêmeas).

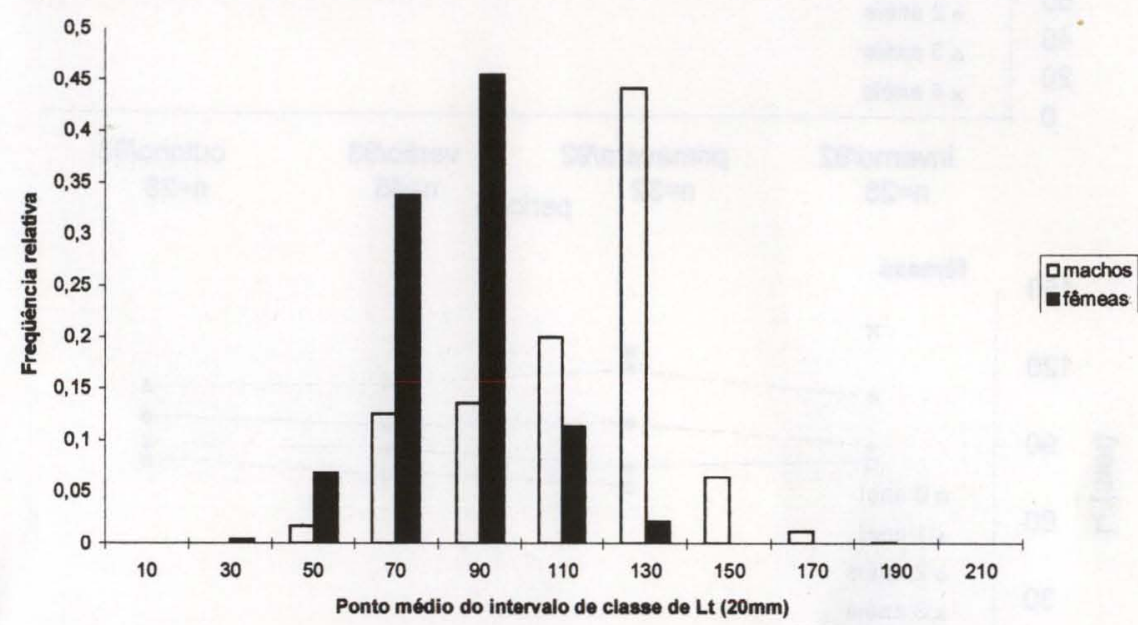

Fig. 1. Distribuição da freqüência relativa das classes de comprimento total, em $20 \mathrm{~mm}$, para machos e fêmeas de Gymnogeophagus lacustris coletados na lagoa Caconde, Rio Grande do Sul, entre os meses de julho/92 a junho/93.

A relação peso x comprimento foi obtida para todo o período estudado. Após a transformação logarítmica das variáveis, obteve-se as seguintes expressões:

Machos: $\mathrm{Wt}=\left(1,3918 \cdot 10^{-5}\right) \cdot\left(\mathrm{Lt}^{3,0466}\right)$

Fêmeas: $\mathrm{Wt}=\left(1,1358 \cdot 10^{-5}\right) \cdot\left(\mathrm{Lt}^{3,0884}\right)$.

Estas relações foram testadas quanto à isometria, através do teste $t$, indicando que as constantes quanto ao tipo de crescimento $(\theta)$ foram estatisticamente diferentes de $3(\mathrm{p}<0,05)$, para ambos os sexos.

Tanto machos quanto fêmeas apresentaram quatro anéis em suas escamas, sendo que estes formam-se entre os meses de inverno e primavera (Fig. 2). A periodicidade na formação dos anéis etários foi confirmada pelos resultados da transformação Ford-Walford:

Machos: $\mathrm{Lt}+\Delta \mathrm{t}=17,182+0,91684 . \mathrm{Lt}(\mathrm{r}=0,98)$

Fêmeas: $\mathrm{Lt}+\Delta \mathrm{t}=11,381+0,90535$. $\mathrm{Lt}(\mathrm{r}=0,93)$.

A correção da idade relativa estimou a época de formação do primeiro anel etário:

Machos: $\mathrm{Lt}^{*}=-0,46145-0,32096 . \mathrm{t}^{*}(\mathrm{r}=0,97)$

Fêmeas: $\mathrm{Lt}^{*}=-0,73884-0,47867 . \mathrm{t}^{*}(\mathrm{r}=0,94)$. 

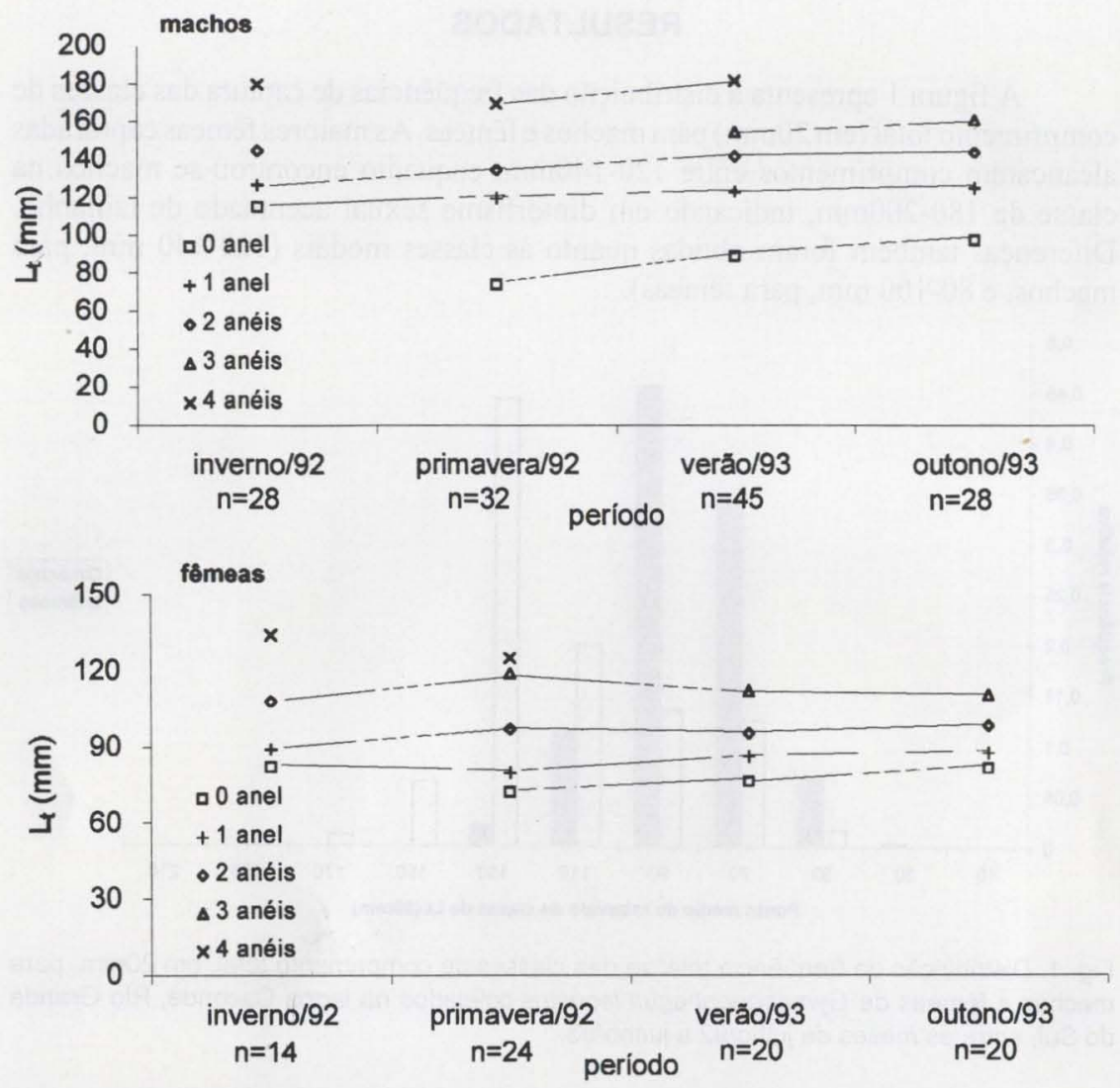

Fig. 2. Valores médios dos comprimentos totais com o mesmo número de anéis plotados contra as estações do ano para Gymnogeophagus lacustris na lagoa Caconde, Rio Grande do Sul, entre os meses de julho/92 a junho/93.

O primeiro anel etário forma-se entre o primeiro e o segundo ano de vida dos indivíduos ( $\mathrm{t}_{\mathrm{l}}=1,43$ anos para os machos e $\mathrm{t}_{1}=1,54$ anos para as fêmeas).

As curvas de crescimento em comprimento total (Fig. 3) confirmam o acentuado dimorfismo sexual quanto ao tamanho para a espécie, com machos alcançando comprimentos médios máximos bem maiores do que as fêmeas. Estas, porém, crescem mais rapidamente e são tão longevas quanto os machos. O dimorfismo sexual também está refletido nas curvas de crescimento em peso (Fig. 4).

\section{DISCUSSÃO}

Estudos existentes sobre o crescimento de ciclídeos no Brasil concentram-se nas espécies Geophagus brasiliensis (Quoy \& Gaimard, 1824) (BARBIERI et al. 1980), Cichlasoma bimaculatus (Linnaeus, 1758) (NomURA \& BARBOSA 1980) e 


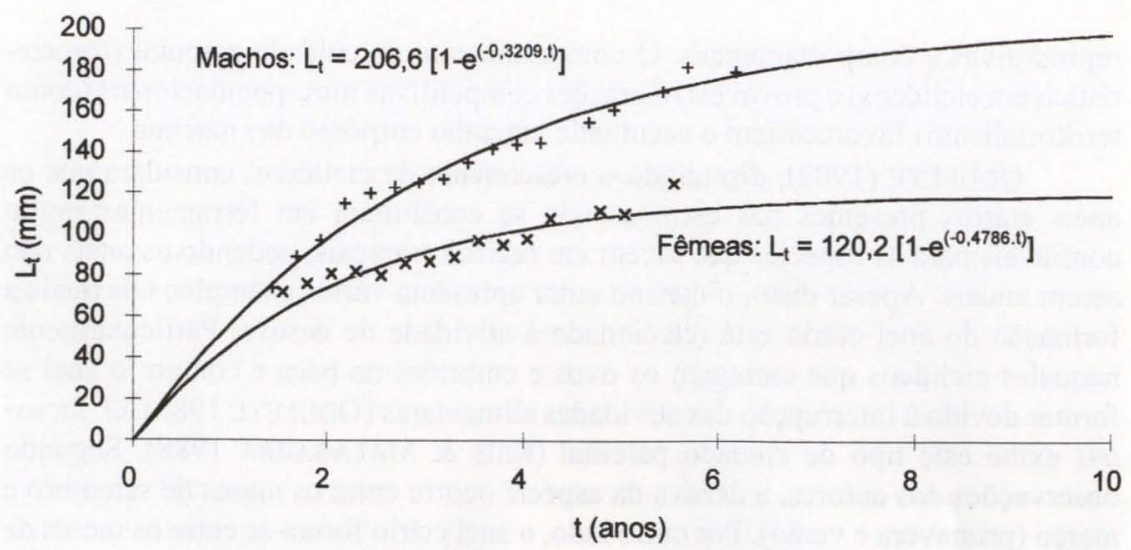

Fig. 3. Curvas de crescimento em comprimento total para Gymnogeophagus lacustris na lagoa Caconde, Rio Grande do Sul, entre os meses de julho/92 a junho/93.

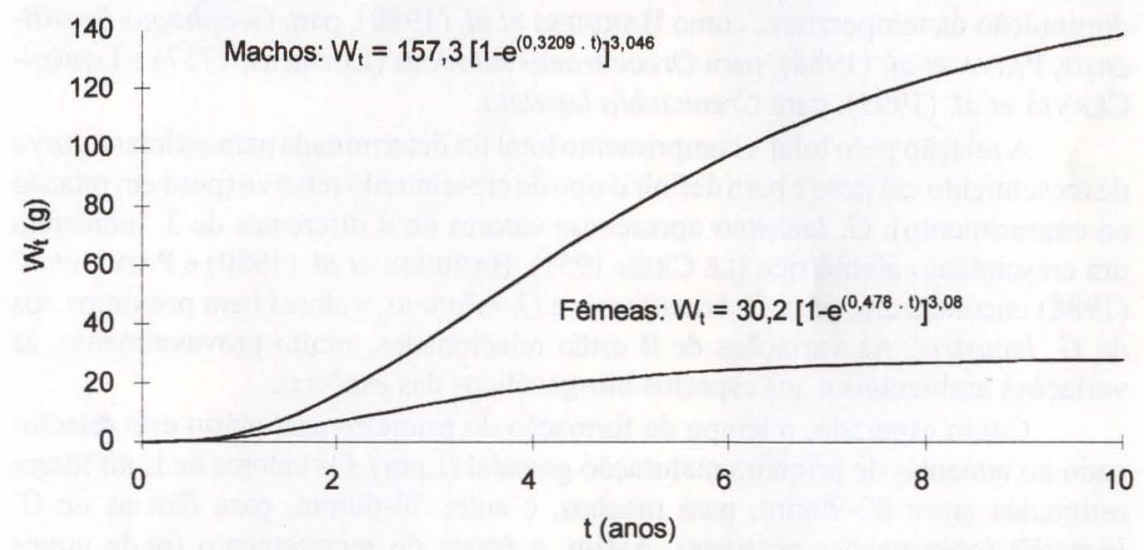

Fig. 4. Curvas de crescimento em peso total para Gymnogeophagus lacustris na lagoa Caconde, Rio Grande do Sul, entre os meses de julho/92 a junho/93.

Crenicichla lepidota Heckel, 1840 (LOBÓN-CERVIÁ et al. 1993), além dos trabalhos realizados com diversas espécies de tilápias, tanto em ambientes naturais ou confinados (BARBIERI et al. 1986; PAIVA et al. 1988, entre outros). Todos estes trabalhos demonstraram que os machos alcançam comprimentos maiores do que as fêmeas, podendo ser característica dos ciclídeos. No entanto, em nenhum deles o dimorfismo foi tão acentuado quanto o observado em $G$. lacustris. Talvez esta diferença possa ser reflexo de um gasto energético diferenciado entre os sexos, com as fêmeas alocando mais energia à reprodução e, assim, alcançando comprimentos assintóticos menores. Por outro lado, esta consideração não valeria de todo, pois em outras espécies de peixes, as fêmeas também apresentam um gasto energético acentuado e, mesmo assim, são maiores do que os machos (principalmente em Characiformes). Estas relações devem ser vistas sempre à luz das estratégias 
reprodutivas e comportamentais. O comportamento de cuidado parental (característico em ciclídeos) e prováveis interações competitivas intra-populacionais (como territorialismo) favoreceriam o acentuado tamanho corpóreo dos machos.

ODULEYE (1982), discutindo o crescimento de ciclídeos, considera que os anéis etários presentes nas escamas não se constituem em ferramentas muito confiáveis para as espécies que vivem em regiões tropicais, podendo os anéis não serem anuais. Apesar disto, o mesmo autor apresenta vários exemplos nos quais a formação do anel etário está relacionado à atividade de desova. Particularmente naqueles ciclídeos que carregam os ovos e embriões na boca é comum o anel se formar devido à interrupção das atividades alimentares (ODULEYE 1982). G. lacustris exibe este tipo de cuidado parental (ReIS \& Malabarba 1988). Segundo observações dos autores, a desova da espécie ocorre entre os meses de setembro e março (primavera e verão). Por outro lado, o anel etário forma-se entre os meses de inverno e primavera, relacionando-se mais à maturação gonadal e às condições ambientais (temperatura) do que ao comportamento de cuidado da prole. Outros autores também relatam que a formação do anel etário se forma em decorrência da diminuição da temperatura, como BARBIERI et al. (1980), para Geophagus brasiliensis, PAIVA et al. (1988), para Oreochromis niloticus (Linnaeus, 1757) e LoBóNCERVIÁ et al. (1993), para Crenicichla lepidota.

A relação peso total $x$ comprimento total foi determinada para estimar a curva de crescimento em peso e para definir o tipo de crescimento relativo (peso em relação ao comprimento). G. lacustris apresentou valores de $\theta$ diferentes de 3 , indicando um crescimento alométrico (LE CREN 1951). BARBIERI et al. (1980) e PAIVA et al. (1988) encontraram, para G. brasiliensis e $O$. niloticus, valores bem próximos aos de $G$. lacustris. As variações de $\theta$ estão relacionadas, muito provavelmente, às variações ambientais e aos aspectos bio-genéticos das espécies.

Como esperado, o tempo de formação do primeiro anel etário está relacionado ao tamanho de primeira maturação gonadal (Lpm). Os valores de Lpm foram estimados entre $80-90 \mathrm{~mm}$, para machos, e entre $50-60 \mathrm{~mm}$, para fêmeas de $G$. lacustris (observações pessoais). Assim, a época de recrutamento (onde novos indivíduos são incorporados às capturas) ocorre entre o final do verão até meados do outono. Estes animais se reproduzirão pela primeira vez no verão do ano seguinte, com aproximadamente um ano e meio de idade (pois nasceram na primavera e verão do ano anterior).

LIZAMA \& VAZZOLER (1993) demonstraram uma nítida relação inversa entre os comprimentos médios máximos $(\mathrm{L} \propto)$ e a taxa de crescimento $(\mathrm{k})$. Os valores estimados de k neste estudo demonstraram que os machos de G. lacustris crescem mais lentamente $(k=0,32)$ do que as fềmeas $(k=0,47)$, alcançando comprimentos assintóticos bem maiores ( $\mathrm{L} \propto=206,6 \mathrm{~mm})$. Esta mesma tendência foi observada por BARBIERI et al. (1980), para Geophagus brasiliensis e por BARBIERI et al. (1986) para Tilapia rendalli. Considera-se que as estimativas de L $\propto$, feitas para G. lacustris, foram satisfatórias se comparadas aos dados de frequeência de captura dos exemplares, apresentados na figura 1. Em relação à biomassa, a espécie atinge pesos médios máximos menores que outros ciclídeos estudados, independente de Lt, reflexo provável de sua alometria. 


\section{CONCLUSÕES}

Podemos concluir que o crescimento de Gymnogeophagus lacustris, na lagoa Caconde, Rio Grande do Sul, caracteriza-se por um acentuado dimorfismo sexual. Tanto fêmeas quanto machos apresentam o mesmo número de anéis em suas escamas, porém os machos são maiores, para uma mesma idade, e crescem mais lentamente do que as fêmeas. Para ambos os sexos, o anel etário começa a ser formado entre o primeiro e segundo anos de vida e sua formação está relacionada, principalmente, à maturação gonadal e à queda dos valores de temperatura da água.

\section{REFERÊNCIAS BIBLIOGRÁFICAS}

Barbieri, G.; J.A. Pereira \& F.J.C. Branco Costa. 1980. Crescimento de Geophagus brasiliensis (Quoy \& Gaimard, 1824) (Pisces, Cichlidae) pelo método do retrocálculo. B. Núcl. Est.-s. Ci-s. Mar., Maceió, 4: 9-32.

Barbieri, G.; M.C. Barbieri; M.A. Marins; J.R. Verani; A.C. Peret \& J.A. PEREIRA. 1986. Crecimiento de Tilapia rendalli (Boulenger, 1896) en el embalse del Monjolinho, São Carlos, Estado de São Paulo, Brasil. Rev. Lat. Acui., Lima, 27: 18-28.

BertalanfFy, L. VON. 1938. A quantitative theory of organic growth. Human Biol., Berlin, 10 (2): 181-213.

LE CREN, E.D. 1951. The length-weight relationship and seasonal cycle in gonad weight and condition in the perch (Percafluviatilis). Jour. Anim. Ecol., Oxford, 20: 201-209.

LizAma, M. DE L. A. \& A.E.A. DE M. VAzZoler. 1993. Crescimento em peixes do Brasil: uma síntese comentada. Revista Unimar, Maringá, 15 (Supl.): 141-173.

Lobón-CerviÁ, J.; C.G. Utrilla; E. Queirol \& M.A. Puig. 1993. Population ecology of pike-cichlid, Crenicichla lepidota, in two streams of the Brazilian Pampa subject to a severa drought. Jour. Fish Biol., London, 43: 537-557.

MALABARBA, L.R. \& E.A. IsAiA. 1992. The fresh-water fish fauna of the Rio Tramandaí Drainage, Rio Grande do Sul, Brazil, with a discussion of its historical origin. Comun. Mus. Ciênc. PUCRS, sér. zool., Porto Alegre, 5 (12): 197-223.

NomURA, H. \& J.M. BARBoSA. 1980. Biologia do acará-cascudo, Cichlasoma bimaculatum (L., 1758) do Riacho Bem Posta (Campo Maior, Piauí) (Osteichthyes, Cichlidae). Rev. Brasil. Biol., Rio de Janeiro, 40 (1): 159-163.

ODULEYE, S.O. 1982. Growth and growth regulation in the cichlids. Aquaculture, Amsterdan, 27 (3): 301-306.

PaiVA, P. DE; J.R. Verani; C.S.R. MAInARdes-PinTo \& Y.A. TABATA. 1988. Studies on the growth and reproduction in the cichlid Oreochromis niloticus (Linnaeus, 1757) in a earthern pond. Bol. Inst. Pesca, São Paulo, 15 (2): 109-114.

ReIS, R. \& L.R. Malabarba. 1988. Revision of the Neotropical Cichlid Genus Gymnogeophagus, Ribeiro, 1918, with descriptions of two new species (Pisces, Perciformes). Revta bras. Zool., São Paulo, 4 (4): 259-305.

SANTOS, E.P. DOS. 1978. Dinâmica de populações aplicada à pesca e piscicultu- 
ra. São Paulo, Hucitec-Edusp, 129p.

WALFORD, L.A. 1946. A new graphic method of describing the growth of animals.

Biol. Bull. mar. Biol. Lab., Woods Hole, 90 (1): 141-147.

Recebido em 17.IV.1997; aceito em 24.VII.1998. 